Publicacions Matemàtiques, Vol 36 (1992), 131-155.

\title{
FINSLER METRICS WITH PROPERTIES \\ OF THE KOBAYASHI METRIC ON CONVEX DOMAINS
}

\author{
MYUNG-YULL PANG
}

\begin{abstract}
The structure of complex Finsler manifolds is studied when the Finsler metric has the property of the Kobayashi metric on convex domains: (real) geodesics localiy extend to complex curves (extremal disks). It is shown that this property of the Finsler metric induces a complex foliation of the cotangent space closely related to geodesics. Each geodesic of the metric is then shown to have a unique extension to a maximal totally geodesic complex curve $\Sigma$ which has properties of extremal disks. Under the additional conditions that the metric is complete and the holomorphic sectional curvature is $-4, \Sigma$ coincides with an extremal disk and a theorem of Faran is recovered: the Finsler metric coincides with the Kobayashi metric.
\end{abstract}

\section{Introduction}

The Riemann mapping theorem says that all simply connected domains in $\mathbb{C}$ different from $\mathbb{C}$ are biholomorphically equivalent. It is a well known fact that this theorem does not hold for domains in $\mathbb{C}^{n}$ for $n>1$, and the classification of bounded domains up to biholomorphism has been an important problem in several complex variables. One approach to understanding the structure of bounded domains is to study biholomorphically invariant metrics such as the Kobayashi or Carathéodory metrics [K]] [BD] [L3] [Pa]. In [L1] and [L2], Lempert showed that these metrics are extremely well-behaved in the special case when the domain is strictly linearly convex and has smooth boundary: In this case, the two metrics coincide, and the infinitesimal form $F_{K}$ of the Kobayashi metric falls into a special class of smooth Finsler metrics with constant holomorphic sectional curvature $K=-4$. Since the notion of a strictly linearly convex domain is not a biholomorphically invariant concept, it 
is natural to ask how far Lempert's results can be extended to a more general (biholomorphically invariant) complex manifolds.

One approach to this problem is to study $F_{K}$ from a more invariant point of view. The first step is to characterize the properties of an abstract Finsler metric $F$ on an abstract complex manifold $M^{n}$ which are necessary for Lempert's results to hold. A second, and more difficult, step is to determine when the Kobayashi metric of a bounded domain in $\mathbb{C}^{n}$ has these properties. In $[\mathbf{F}]$, Faran analyzed the local structure of (complex) Finsler manifolds and obtained a set of local invariants by applying Cartan's method of equivalence. He proved that vanishing of certain local invariants forces $F$ to coincide with the Kobayashi metric of the underlying manifold $M$ provided that $F$ is a complete metric with $K=-4$. However, from the complex process of constructing these local invariants it is not easy to see how these invariants naturally arise from the properties of Kobayashi metrics obtained from Lempert's work. Thus, one would like to formulate a somewhat more direct description of the local structure that is intuitively more appealing. In this paper, we give such description from the point of view of the calculus of variations by examining the local properties of the Kobayashi rnetric on strictly linearly corvex domains, and derive equivalent conditions to the vanishing of the Faran's invariants from a simple property of Kobayashi metric (Property 1.3).

In order to describe the local structure of the Kobayashi metric, we give bricf review of Lempert's work. We define the infinitesimal Kobayashi metric $F_{K}$ on a complex manifold $M$ as follows: For each $v \in T_{x} M$, $x \in M$, let $f$ be a holomorphic map from the unit disk $\Delta \subset \mathbb{C}$ into $M$ such that $f(0)=x$ and $f^{\prime}(0)=\lambda_{f} v$ for $\lambda_{f}>0$. The magnitude $F_{K}(v)$ of $v$ with respect to the infinitesimal Kobayashi metric $F_{K}$ is defined to be the infimum of $\frac{1}{\lambda_{f}}$, where the infirmum is taken over all such $f$. If $f$ actually attains the infimum (i.e. $F_{K}(v)=\frac{1}{\lambda_{f}}$ ), then $f$ is called extremal. It can be casily scen that the metric $F_{K}$ is invariant under the action of the group of biholomorphisms of $M$. Lempert showed that, if $M=D \subset \mathbb{C}^{n}$ is a bounded strictly linearly convex domain with smooth boundary, then $F_{K}$ is a smooth complex Finsler metric $[\mathbf{L 1}]$, [L2], i.e. $F_{K}$ is smooth outside the zero section of $T D$ and satisfies the following conditions:

$F_{K}(v)>0$ for $v \neq 0, \quad F_{K}(z \cdot v)=|z| F_{K}(v)$ for $z \in \mathbb{C}$, and (1.2)

$F_{K}\left(v_{1}+v_{2}\right) \leq F_{K}\left(v_{1}\right)+F_{K}\left(v_{2}\right) \quad$ for $v_{1}, v_{2} \in T_{x} D, \quad x \in D$,

where equality in (1.2) holds only when $v_{1}$ and $v_{2}$ are colinear. Moreover, 
he proved the following theorem:

Theorem (Lempert). Suppose that $D$ is a bounded strictly linearly convex domain with smooth boundary.

(1) There is a unique extremal map corresponding to each $v \in T D$.

(2) All the extremal maps are proper isometric imbeddings, and can be smoothly extended to the closed unit disk $\bar{\Delta}$.

(3) The extremal disks $f(\Delta)$ passing through a point $x \in D$ form a complex foliation of $D-\{x\}$.

(4) Extremal disks are (the only) one-dimensional holomorphic retracts of $D$.

One of the key ideas in describing the geometry of $D$ is the construction of the holomorphic retract of $D$ onto the extremal disk $f(\Delta)$. Lempert proved that the field of holomorphic tangent planes of $\partial D$ on $f(\partial \Delta)$ can be holomorphically extended to the interior of the disk $f(\Delta)$, and defines a holomorphic field of complex hyperplanes on $f(\Delta)$ that are transversal to $f(\Delta)$. In other words, there is a well defined $(n-1)$-dimensional holomorphic vector bundle $p: E \rightarrow f(\Delta)$ over the extremal disk with fibers defined by the hyperplanes in $\mathbb{C}^{n}$. The union of the hyperplanes contains the domain $D$, and the holomorphic retract is defined by the restriction to $D$ of the projection map $p$.

The existence of such holomorphic retracts has further implications. For example, it forces every (locally length minimizing, connected) geodesic curve of $F_{K}$ to be contained in an extremal disk. The properties of the Kobayashi metric that interests us are the following:

Corollary. Let $f: \Delta \rightarrow D$ be a extremal map for $v \in T_{x} D$.

(1.3) The extremal disk $f(\Delta)$ coincides with the union of geodesic curves through $x$ tangent to a common complex line in $T_{x} M$.

(1.4) There is a canonical splitting $T D_{\mid f(\Delta)}=T(f(\Delta)) \oplus E$ where $E$ is an $(n-1)$-dimensional holomorphic subbundle of the restriction $T D_{\mid f(\Delta)}$ of the tangent bundle TD to $f(\Delta)$.

We wish to generalize the condition (1.3) to an abstract complex Finsler metric $F$ defined on an $n$-dimensional complex manifold $M$. Let exp denote the exponential map from a neighborhood of $0 \in T_{x} M$ into $M$ defined by the geodesics of $F$. For each tangent vector $v \in T_{x} M$, the image $\exp \left(U_{v}\right)$ of a small neighborhood $U_{v}$ of 0 in the complex line $\mathbb{C} \cdot v$ defines a surface in $M$. A reasonable generalization of the condition (1.3) is the following:

(1.5) For all $v \in T M$, the surface $\exp \left(U_{v}\right)$ is a complex curve (1dimensional complex submanifold) in $M$. 
This condition was first introduced by Royden [R], and it is, in fact, equivalent to a condition given by Faran $[\mathbf{F}]$.

The purpose of this paper is to study the local structure on of Finsler rnetrics satisfying condition (1.5), and further, to show that many properties of the Kobayashi metric of convex domains extend to this more general class of complex manifolds. One of our major results is a construction of a biholomorphically invariant family of complex curves which enjoys many of the properties of extremal disks in convex domains. To see how such complex curves are constructed, recall, from the calculus of variations, that the metric $F$ uniquely determines a vector field $X$ on the cotangent space of $M$, called the geodesic vector field, such that the integral curves of $X$ are mapped into geodesics of $F$ by the projection map $\pi: T^{*} M \longrightarrow M$. Let $Z$ be the vector field on $T_{0}^{*} M=\left\{v \in T^{*} M \mid v \neq 0\right\}$ generated by the circle action of unimodular complex numbers defined by multiplication on $T_{0}^{*} M$.

Theorem A. The following conditions are equivalent (Theorem 4.7):

1. The surface $\exp \left(U_{v}\right) \subset M$ is a complex curve for all $v \in T M$.

2. $[X, J X]=\kappa Z$ for some smooth function $\kappa$ on $T_{0}^{*} M$.

3. The distribution $\mathcal{D}=\mathbb{C} X \oplus \mathbb{C} Z \subset T\left(T_{0}^{*} M\right)$ is involutive.

If any of the above conditions is satisfied then each complex curve $\exp \left(U_{v}\right)$ extends uniquely to a maximal, totally geodesic, immersed complex curve $\Sigma \hookrightarrow M$ (Theorem 4.9).

The significance of the condition 2 and 3 in the thcorem is as follows: The condition 2 provides a computational methods to check whether $F$ satisfies the property (1.5). The condition 3 implies that, by Frobenius Theorem, the distribution $\mathcal{D}$ defines a 2-dimensional complex foliation $\mathcal{F}_{0}$ of $T_{0}^{*} M$. The complex curve $\Sigma$ is constructed by projecting each leaf of the foliation $\mathcal{F}_{0}$ by the projection map $\pi$ onto $M$.

The curves $\Sigma$ share many local properties in common with the extremal disks described in the Lempert's theorem. For examplc, by Theorem A, any real gcodesic curve of $F$ is contained in one of the curves $\Sigma$. Furthermore, a generalization of the property (3) in Lempert's theorem holds: the complex curves $\sum$ passing through a point $x$ form a complex foliation of some neighborhood of $x$. A less trivial result is the following generalization of property (1.4):

Theorem B. For each complex curve $\Sigma$, there is a cononical splitting $T M_{\mid \Sigma}=T \Sigma \oplus T^{\perp} \Sigma$, where $T^{\perp} \Sigma$ is an $(n-1)$-dimensional holomorphic subbundle of $T M_{\mid \Sigma}$. (Theorem 4.9.) 
The significance of the function $\kappa$ in the Theorem $A$ is its relation to the holomorphic sectional curvature of $F$. Note that cach complex curve in $M$ is naturally equipped with a Hernitian metric induced by $F$, and, therefore, has an associatcd Gaussian curvature. Following the definition by Wong and Royden $[\mathbf{W}][\mathbf{R}]$, we define the holomorphic sectional curvature of $F$ at $v$ by the Gaussian curvature of the curve $\exp \left(U_{v}\right)$

Theorem C. If $F$ is a complex Finsler metric satisfying the condition (1.5) then the holomorphic sectional curvature of $F$ is determined by the function $\kappa$.

Finally, using the result described above, we show that, under the condition that. $F$ is complete and $\kappa=-4$ the complex curves $\Sigma$ coincide with the extremal disks. This result was proved earlicr by Faran [F]. Notc that, from Lempert's result, the Kobayashi metric $F_{K}$ on a strictly linearly convex domain $D \subset \mathbb{C}^{n}$ has constant holomorphic sectional curvature -4 . (This is a direct consequence of the fact that every extremal map $f: \Delta \rightarrow D$ is an isometry with respect to the Poincaré metric and $F_{K}$ such that $f(\Delta)$ is locally defined by $\exp \left(U_{n}\right)$.)

4.24 Theorem $[\mathbf{F}]$. Suppose $F$ is a complete complex Finsler metric on a complex manifold $M$ with constant holomorphic sectional curvature -4 satisfying the property (1.5). Then $F=F_{K}$, where $F_{K}$ is the Kobayashi metric on $M$.

The papcr is organized as follows: In Section 2, we develop basic tools and prove some basic facts about complex Finsler manifolds. Section 3 is an introduction of Legendre foliations and its application to complex Finsler manifolds. In Section 4, we prove the main theorem using the results of Sections 2 and 3.

Throughout the paper, $M$ denotes an $n$ dimensional complex manifold and $F$ a complex Finsler metric on $M$. The following notations are used:

(1) The indices $a, b$ and $c$ range from 1 through $2 n$, and $\alpha, \beta, \gamma$ range from 1 through $2 n-1$. Summation conventions are in force throughout.

(2) $\left(x^{1}, \ldots, x^{n}, x^{n+1}, \ldots, x^{2 n}\right)$ denote the real coordinates on $M$ obtained from a holomorphic coordinates $x^{\nu}+i x^{n+\nu}$ for $\nu=1, \ldots, n$.

(3) $\left(x^{1}, \ldots, x^{n}, x^{n+1}, \ldots, x^{2 n} ; u^{1}, \ldots, u^{n}, u^{n+1}, \ldots, u^{2 n}\right)$ denote the coordinates on $T^{*} M$ induced by $\left(x^{1}, \ldots, x^{n}, x^{n+1}, \ldots, x^{2 n}\right)$.

(4) For $F \in C^{\infty}\left(T^{*} M\right), F_{a}, F_{a b}, \ldots$ denote $\frac{\partial F}{\partial u^{a}}, \frac{\partial^{2} F^{2}}{\partial u^{a} j u^{u}}$ and so on.

(5) If $V$ is a vector field on a manifold $M, e^{t V}: M \rightarrow M$ dentotes the 1-parameter farnily of diffeomorphisms generated by $V$. Thus, for 
cach $x \in M, t \mapsto e^{t V} x$ is an integral curve of $V$ starting at $x$ (i.e. $e^{0 V} x=x$ and $\frac{d}{d t} \mid e^{t V} x=V_{e^{t V}}$ ).

Acknowledgments. I would like to thank T. Duchamp for his help and encouragement, and for introducing me to this subject. I also wish to express my thanks to J. Bland for conversations.

\section{Complex Finsler Metrics}

In this section, we prove some general facts about complex Finsler metrics. A complex Finsler metric on the cotangent bundle of $M$ is a map $F: T^{*} M \longrightarrow \mathbb{R}$ satisfying properties (1.1) and (1.2). When $M$ is equipped with a complex Finsler metric, we will call $M$ a complex Finsler manifold.

2.1. The Geodesic Vector Field and Complex Structure. In order to define the exponential map, we introdwe the geodesic vector ficld on $T^{*} M$. Recall that $T^{*} M$ is naturally cquipped with a 1 -form $\zeta$ defined by the equation

$$
\zeta=\sum_{a=1}^{2 n} u^{a} d x^{a}
$$

and that the 2-form $d \zeta$ is a symplectic 2-form on $T^{*} M$ (i.e. a smooth closed 2 -form on $T^{*} M$ satisfying the non-degeneracy condition $(d \zeta)^{2 n} \neq$ 0 ). The geodesic vector field $X$ on $T^{*} M$ is uniquely determined by the condition

$$
X \unlhd d \zeta=-F d F
$$

In particular, the identity $X F=0$ holds. In terms of coordinates, we have

$$
X=F \sum_{a=1}^{2 n}\left\{F_{a} \frac{\partial}{\partial x^{\alpha}}-\frac{\partial F}{\partial x^{\alpha}} \frac{\partial}{\partial u^{\alpha}}\right\}
$$

To describe how $X$ is related to the complex structure, consider coordinate expression of the complex structure on $T^{*} M$. The complex structure $J$ on $M$ is cxpressed as

$$
J \frac{\partial}{\partial x^{\alpha}}=J_{a}^{b} \frac{\partial}{\partial x^{b}} \quad \text { where } \quad\left(J_{b}^{a}\right)=\left(\begin{array}{cc}
0 & -I \\
I & 0
\end{array}\right)
$$


and $I$ is the $(n \times n)$-identity matrix. The natural complex structure on $T^{*} M$ is then given by

$$
J \frac{\partial}{\partial x^{a}}=J_{a}^{b} \frac{\partial}{\partial x^{b}}, \quad J \frac{\partial}{\partial u^{a}}=-J_{a}^{b} \frac{\partial}{\partial u^{b}} .
$$

It can be directly checked that the complex structure defined by this is independent of the choice of coordinates. By abuse of notation, we will denote this complex structure on $T^{*} M$ by $J$. From the definition above, it is clcar that $J \circ \pi_{*}=\pi_{*} \circ J$.

Note that condition (1.1) provides a compatibility condition of $F$ and the complex structure, which can be expressed as follows: Let $Y$ be the radial vector field on $T_{0}^{*} M$ generated by the action of $R$ by rmultiplication of $e^{t}, t \in \mathbb{R}$. It can be easily ehecked that $Y$ and $Z$ satisfy the relation

$$
Z=J Y
$$

Thus, if $F$ satisfics the condition (1.1), we have $F\left(e^{s i} v\right)=F(v)$ and $F\left(e^{t} v\right)=e^{t} F(v)$, and therefore the following identities hold:

$$
Z F=0, \quad Y F=F .
$$

Note that the coodinate cxpressions of $Y$ and $Z$ are

$$
Y=u^{a} \frac{\partial}{\partial u^{a}}, \quad Z=-J_{b}^{a} u^{b} \frac{\partial}{\partial u^{a}} .
$$

Therefore, conditions (2.8) are equivalent to

$$
J_{b}^{a} u^{b} F_{t 2}=0, \quad F_{i 2} u^{a}=F .
$$

2.11 Lemma. The follouning identities are sotisfied:

$$
\begin{array}{ll}
{[Z, X]=-J X,} & {[Z, J X]=X,} \\
{[Y, X]=X,} & {[Y, J X]=J X, \quad[Y, Z]=0 .}
\end{array}
$$

Proof: The computations in the proof of this lemma are based on the identities (2.8)-(2.10) and the following basic identities derived from them:

$$
\left[Y, \frac{\partial}{\partial x^{u}}\right]=\left[Z, \frac{\partial}{\partial x^{u}}\right]=0, \quad\left[Y, \frac{\partial}{\partial u^{\alpha}}\right]=-\frac{\partial}{\partial u^{2}}, \quad\left[Z, \frac{\partial}{\partial u^{a}}\right]=J_{a}^{b} \frac{\partial}{\partial u^{b}}
$$


Now compute $[Z, X]$ using coordinates:

$$
\begin{aligned}
{[Z, X]=\mathcal{L}_{Z} X } & =\mathcal{L}_{Z}\left\{F \sum_{a=1}^{2 n}\left(F_{a} \frac{\partial}{\partial x^{a}}-\frac{\partial F}{\partial x^{a}} \frac{\partial}{\partial u^{a}}\right)\right\} \\
& =F \sum_{a=1}^{2 n}\left\{\left(Z F_{a}\right) \frac{\partial}{\partial x^{a}}-\frac{\partial F}{\partial x^{a}} \mathcal{L}_{Z}\left(\frac{\partial}{\partial u^{a}}\right)\right\} .
\end{aligned}
$$

Observe that

$$
\left(Z F_{a}\right)=Z\left(\frac{\partial F}{\partial u^{a}}\right)=\frac{\partial(Z F)}{\partial u^{a}}+\left[Z, \frac{\partial}{\partial u^{a}}\right] F=\left(J_{a}^{b} \frac{\partial}{\partial u^{b}}\right) F=J_{a}^{b} F_{b} .
$$

Therefore, using the skew-symmetry of $J_{b}^{a}$, we obtain

$$
\begin{aligned}
{[Z, X] } & =-F \sum_{a=1}^{2 n}\left(J_{b}^{a} F_{b} \frac{\partial}{\partial x^{a}}+J_{a}^{b} \frac{\partial F}{\partial x^{a}} \frac{\partial}{\partial u^{b}}\right) \\
& =-J\left\{F \sum_{a=1}^{2 n}\left(F_{a} \frac{\partial}{\partial x^{a}}-\frac{\partial F}{\partial x^{a}} \frac{\partial}{\partial u^{a}}\right)\right\}=-J X .
\end{aligned}
$$

To show the identity $[Z, J X]=X$, note that $\mathcal{L}_{Z} J=0$, and compute

$$
[Z, J X]=\mathcal{L}_{Z}(J X)=J\left(\mathcal{L}_{Z} X\right)=J(-J X)=X .
$$

To prove the identity $[Y, X]=X$, note that

$$
Y F_{a \mathrm{a}}=Y\left(\frac{\partial F}{\partial u^{2}}\right)=\frac{\partial}{\partial u^{a}}(Y F)+\left[Y, \frac{\partial}{\partial u^{a}}\right] F=F_{a}-F_{a}=0 .
$$

Using this identity, compute $[Y, X]$ as follows:

$$
\begin{aligned}
{[Y, X]=} & \mathcal{L}_{Y} X=\mathcal{L}_{Y}\left\{F \sum_{a=1}^{2 n}\left(F_{a} \frac{\partial}{\partial x^{a}}-\frac{\partial F}{\partial x^{a}} \frac{\partial}{\partial u^{a}}\right)\right\} \\
= & (Y F) \sum_{a=1}^{2 n}\left(F_{a} \frac{\partial}{\partial x^{a}}-\frac{\partial F}{\partial x^{a}} \frac{\partial}{\partial u^{a}}\right) \\
& -F \mathcal{L}_{Y}\left\{\sum_{a=1}^{2 n}\left(F_{a} \frac{\partial}{\partial x^{a}}-\frac{\partial F}{\partial x^{a}} \frac{\partial}{\partial u^{a}}\right)\right\} \\
= & F \sum_{a=1}^{2 n}\left(F_{a} \frac{\partial}{\partial x^{a}}-\frac{\partial F}{\partial x^{a}} \frac{\partial}{\partial u^{a}}\right) \\
& -F \sum_{a=1}^{2 n}\left\{Y\left(\frac{\partial F}{\partial x^{a}}\right) \frac{\partial}{\partial u^{a}}+\frac{\partial F}{\partial x^{a}}\left(\mathcal{L}_{Y} \frac{\partial}{\partial u^{a}}\right)\right\} \\
= & X-\sum_{a=1}^{2 n}\left\{\left(\frac{\partial(Y F)}{\partial x^{a}}\right) \frac{\partial}{\partial u^{a}}-\frac{\partial F}{\partial x^{a}} \frac{\partial}{\partial u^{a}}\right\}=X .
\end{aligned}
$$


The identity $[Y, Z]=0$ is clear since the actions of $e^{t}$ and $e^{s i}$ commute, and the identity $[Y, J X]=J X$ follows from the computation:

$$
[Y, J X]=\left[Y,-\mathcal{L}_{Z} X\right]=-\mathcal{L}_{Z}[Y, X]+\left[\mathcal{L}_{Z} Y, X\right]=-\mathcal{L}_{Z} X=J X
$$

2.13 Lemma. The vector fields $J X$ and $[X, J X]$ satisfy the identities:

$$
\zeta(J X)=d F(J X)=0, \quad \text { and } \quad \zeta([X, J X])=d F([X, J X])=0 .
$$

In particular, $J X$ and $[X, J X]$ are tangent to the submanifold $S_{F}^{*} M \subset$ $T_{0}^{*} M$.

Proof: To prove the lemma, recall that we have the identities $\mathcal{L}_{X} \varsigma=$ $X\lrcorner d \zeta=-F d F, X F=0$ and $\zeta(Z)=0$. Also, recall from identities (2.8) and (2.12) that $Z F=0$ and $J X=[X, Z]$. Using these identities and the fact that $\mathcal{L}_{X}$ is a derivation, compute as follows:

$\zeta(J X)=\zeta([X, Z])=\zeta\left(\mathcal{L}_{X} Z\right)=\mathcal{L}_{X}(\zeta(Z))-\left(\mathcal{L}_{X} \zeta\right)(Z)=F d F(Z)=0$ $d F(J X)=d F([X, Z])=X Z F-Z X F=0$

Using these identities again, we complete the proof of the lemma:

$$
\begin{aligned}
& \zeta([X, J X])=\zeta\left(\mathcal{L}_{X}(J X)\right)=\mathcal{L}_{X}\{\zeta(J X)\}-\left(\mathcal{L}_{X} \zeta\right)(J X)=F d F(J X)=0 \\
& d F([X, J X])=X(J X) F-(J X) X F=0 .
\end{aligned}
$$

2.15 The Exponential Map. The exponential map is defined similarly as in the case of Hermitian manifolds. To define it, we introduce the dual complex Finsler metric $\tilde{F}: T M \longrightarrow \mathbb{R}$ satisfying conditions (1.1) and (1.2), and define geodesics as curves with locally length minimizing property with respect to $\vec{F}$. We briefly review some concepts of the calculus of variations. For more details about the calculus of variations see [GF] and [S].

To define $\tilde{F}$, let $T_{0} M=\{v \in T M \mid v \neq 0\}$, and define a bundle rnap $\Psi: T_{0}^{*} M \longrightarrow T_{0} M$ by

$$
\Psi(w)=\pi_{*}\left(X_{w}\right) \quad \text { for } \quad w \in T_{0}^{*} M
$$

In coordinates, we have

$$
\Psi_{x}(w)=\left.\sum_{a=1}^{2 n} F(w) F_{a}(w) \frac{\partial}{\partial x^{n}}\right|_{\left(x^{1}, \ldots, x^{n}, x^{n+1}, \ldots, x^{2 n}\right)}
$$


where $w=\left(x^{1}, \ldots, x^{n}, x^{n+1}, \ldots, x^{2 n} ; u^{1}, \ldots, u^{n}, u^{n+1}, \ldots, u^{2 n}\right)$. From (2.17) and conditions (1.1) and (1.2), it can be shown that $\Psi$ is a diffeomorphism with the property that $\Psi(t w)=t \Psi(w)$ for $t>0$. The inverse $\Phi=\Psi^{-1}$ is usually called the Legendre transformation. For example, if $F$ is the norm induccd from the Hermitian metric $g, \Phi: T M \longrightarrow T^{*} M$ defined by $\{\Phi(v)\}(w)=g(v, w)$ for $v, w \in T_{x} M$. We define $\tilde{F}$ by $F \circ \Phi$. It is clear from the following lemma that $\tilde{F}$ is a complex Finsler metric.

2.18 Lemma. The map $\Phi$ has the property: $\Phi(z v)=\bar{z} \Phi(v)$ for $z \in \mathbb{C}$.

Proof: Because the identity $\Psi(t v)=t \Psi(v)$ holds for $t>0$, to prove the lemma, it suffices to verify the identity $\Phi\left(e^{t i} v\right)=e^{-t i} \Phi(v)$. Note that the 1-parameter farnily of diffcomorphisms $e^{i Z}: T_{0}^{*} M \rightarrow T_{0}^{*} M$ of the vector field $Z$ is simply the complex multiplication by $e^{t i}$ (i.e. $\left.e^{t Z_{v}}=e^{i i} \cdot v\right)$. Recall from Lemma 2.11 that $\mathcal{L}_{Z} X=[Z, X]=-J X$. Also note that from the definition of Lie derivative, $\frac{d}{d t}\left(e_{*}^{-t Z} X_{\left(c^{t} z\right.} w\right)=$ $\left.e_{*}^{-t /}\left(\mathcal{L}_{2} X\right)_{\left(\mathrm{c}^{*} \%\right.} w\right)$. Using these identities and the identity $\pi \circ e^{-t Z}=\pi$, we compute

$$
\begin{aligned}
& \frac{d}{d t}\left\{\Psi\left(e^{l i} v\right)\right\}=\frac{d}{d t}\left\{\Psi\left(c^{t Z} v\right)\right\}=\frac{d}{d t}\left\{\pi_{*} X_{\left(e^{t z} v\right)}\right\}=\frac{d}{d t}\left\{\pi_{*} e_{*}^{-t Z} X_{\left\{\mathrm{c}^{t} Z v\right\}}\right\}
\end{aligned}
$$

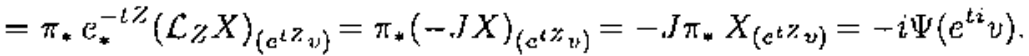

Thercfore, $y(t)=\Psi\left(c^{t \%} v\right)$ satisfies an ordinary difforential equation $y^{\prime}=$ $-i y$ with initial condition $y(0)=\Psi(v)$. From the uniqueness of solution, we can conclude that $\Psi\left(e^{i z} v\right)=e^{-i Z} \Psi(v)$; and from this, it follows that $\Phi\left(e^{t i} v\right)=e^{-t i} \Phi(\nu)$.

A curve $\gamma:\left[t_{1}, t_{2}\right] \rightarrow M$ is called a geodesic if it is a critical curve for the functional

$$
\int_{t_{3}}^{t_{2}} \tilde{F}\left(\frac{d \gamma}{d t}\right) d t, \quad \gamma\left(t_{1}\right), \quad \gamma\left(t_{2}\right) \quad \text { fixed }
$$

Let $v \in T_{x} M$ and $\gamma_{v}:(-\epsilon, \epsilon) \longrightarrow M$ be the geodesic such that $\gamma_{\nu}(0)=x$ and $\frac{d \gamma_{u}}{d t}(0)=v$. As in the case of Hermitian metrics, it can be shown that $\gamma_{\nu}$ is solution of a second order ordinary differential equation (i.e. the Euler-Lagrange equation (GF]), and hence it is uniquely determined. It can be also shown that there is an open neighborhood $N \subset T_{x} M$ of () such that $\gamma_{v}(1)$ is defined for all $v \in N$. Hence, the exponential map $\exp _{x}: N \longrightarrow M$ at $x$ is defined by $\operatorname{cxp}_{x}(v)=\gamma(1)$. From the uniquerıess of solutions of ordinary differential cquations,

$$
\exp _{x}(t v)=\gamma(t)
$$


It is a standard fact from the Calculus of Variations that the projection of the integral curves of $X$ are geodesics on $M$. Hence, it follows that the curve $\gamma(t)=\pi\left(e^{t X} w\right)$ is a geodesic for all $w \in T_{x}^{*} M$ such that $\gamma(0)=x$ and $\frac{d \gamma}{d t}(0)=\pi_{*} X_{w}=\Psi(w)$. Therefore, from the identity $(2.20)$, we obtain

$\exp (t \Psi(w)))=\gamma(t)=\pi\left(e^{t X} w\right)$, or cquivalently, $\exp (t v)=\pi\left(e^{t X} \Phi(v)\right)$ for $v=\Psi(w)$.

2.22 Lemma. For all $v \in T_{0} M$ the exponential map can be expressed in terms of the geodesic vector field $X$ as follows:

$$
\exp \left(t e^{s i} \cdot v\right)=\left\{\pi \circ e^{\imath X} \circ e^{-s Z}\right\}(\Phi(v))
$$

for $s \in \mathbb{R}$ and $t>0$ small.

Proof: Recall from Lemma 2.18 that we have $\Phi\left(e^{s 2} v\right)=e^{-s 2} \Phi(v)$, and $e^{t Z} v=e^{t i} \cdot v$. Using the identity (2.21), we compute

$$
\exp \left(t e^{s i} v\right)=\pi\left(e^{t X} \Phi\left(e^{s i} v\right)\right)=\pi\left(e^{t X} e^{-s i} \Phi(v)\right)=\pi\left(e^{t X} e^{-s z} \Phi(v)\right) .
$$

\section{Legendre Foliations}

In the following, we give a brief introduction to Legendre foliations. Although the theory of Legendre foliations is not required in the statement of the main theorem (Theorem 4.2), it is needed in its proof. For more details about Legendre foliations, we refer the reader to $\{P\}$.

Let $P$ be a smooth $(2 n+1)$-dimensional manifold without boundary with a fixed choice of contact 1-form $\eta$ (i.e. $\eta \wedge(d \eta)^{m} \neq 0$ ). A Legendre foliation is a foliation $\mathcal{F}$ of $P$ by $m$-dimcnsional integral submanifolds of $\eta$. Two Legendre foliations $\mathcal{F}_{1}$ and $\mathcal{F}_{2}$ are said to be equivalent if there is a diffeomorphism $\varphi: P_{1} \longrightarrow P_{2}$ such that $\varphi^{*} \eta_{2}=\eta_{1}$ and $\varphi^{*} \mathcal{F}_{2}=\mathcal{F}_{1}$ where $\varphi^{*} \mathcal{F}_{2}$ is the foliation of $P_{1}$ whose leaves are inverse image of lcaves in $\mathcal{F}_{2}$.

The rclation between Legendre foliations and complex Finsler manifolds can be seen from the following fact $[\mathrm{P}]$ :

The unit cotangent bundle $S_{F}^{*} M=\left\{w \in T^{*} M \mid F(w)=1\right\}$ is equipped with a natural contact 1 -form $\eta$ defined by the pull back. of $\zeta$ to $S_{F}^{*} M$ and the Legendre foliation $\mathcal{F}$ defined by fibers of $\pi: S_{F}^{*} M \longrightarrow M$.

It is shown in $[P]$ that the structure of Legendre foliations defined in this way determines the rnetric $F$ uniquely: 
3.1 Proposition. If $\mathcal{F}_{1}$ and $\mathcal{F}_{2}$ are equivalent Legendre foliations on $S_{F_{1}}^{*} M_{1}$ and $S_{F_{2}}^{*} M_{2}$ where $F_{1}$ and $F_{2}$ are complex Finsler metrics on $M_{1}$ and $M_{2}$ respectively, then $M_{1}$ and $M_{2}$ are isometric as Finsler manifolds (i.e. there exists a diffeomorphism $\varphi: M_{1} \longrightarrow M_{2}$ such that $\left.F_{1}=F_{2} \circ \varphi_{*}\right)$.

To describe the local structure of the Legendre foliation on $S_{F}^{*} M$, let $L$ be the tangent bundle to $\mathcal{F}$. One of the basic local invariants of the Legendre foliation is defined by the restriction to $L$ of the symmetric form

$$
\Pi=F_{a b} d u^{a} \otimes d u^{b}
$$

It is shown in $[\mathbf{P}]$ that, if the triangle inequality (1.2) holds, then $\Pi$ is positive definite, and that there is a canorical reduction of the structure group to $O(2 n-1)$. Note that, from their definitions, the restrictions of vector fields $X$ and $Z$ on $S_{F}^{*} M$ are tangent to $S_{F}^{*} M$, and hence they are invariantly defined vector fields on $S_{F}^{*} M$. If $\left(Z_{\alpha}\right)$ is a local orthonormal frame of $L$ with respect to $\Pi$, there is a local coframe $\left(\theta^{\alpha}, \eta, \xi^{\beta}\right)$ on $S_{F}^{*} M$ such that,

$$
\xi^{\alpha}\left(Z_{\beta}\right)=\delta_{\beta}^{\alpha}, \quad \theta^{\alpha}(X)=\theta^{\alpha}\left(Z_{\beta}\right)=0
$$

satisfying the structure equation:

$$
\begin{aligned}
\left(\begin{array}{c}
d \theta^{\alpha} \\
d \eta \\
d \xi^{\alpha}
\end{array}\right)= & -\left(\begin{array}{ccc}
\pi_{\beta}^{\alpha} & 0 & 0 \\
0 & 0 & 0 \\
0 & 0 & \pi_{\beta}^{\alpha}
\end{array}\right)\left(\begin{array}{c}
\theta^{\beta} \\
\eta \\
\xi^{\beta}
\end{array}\right) \\
& +\left(\begin{array}{c}
-\eta \wedge \xi^{\alpha}+G^{\alpha}{ }_{\beta \gamma} \theta^{\beta} \wedge \xi^{\gamma} \\
\delta_{\beta \gamma} \theta^{\beta} \wedge \xi^{\gamma} \\
R^{\alpha \alpha}{ }_{\beta \gamma} \theta^{\beta} \wedge \theta^{\gamma}+S_{\beta}^{\alpha} \eta \wedge \theta^{\beta}+Q^{\alpha}{ }_{\beta \gamma} \theta^{\beta} \wedge \xi^{\gamma}
\end{array}\right)
\end{aligned}
$$

with the following symmetry conditions:

(i) $G_{\beta \gamma}^{\alpha}, Q_{\beta \gamma}^{\alpha}$ and $S_{\beta}^{\alpha}$ are symmetric in all indices, and

(ii) $R_{\beta \gamma}^{\alpha}=-R^{\alpha}{ }_{\gamma \beta}$ and $\pi_{\beta}^{\alpha}=-\pi_{\alpha}^{\beta}$.

Morcover, $F$ is the induced norm of a Hermitian metric if and only if $G^{\alpha}{ }_{\beta \gamma}=Q^{\alpha x}{ }_{\beta \gamma}=0$. In this case, the tensors $R^{\alpha}{ }_{\beta \gamma}$ and $S_{\beta}^{\alpha}$ are related to the curvature tensor of the Hermitian metric. This can be easily stated in lower dimensional case:

3.5 Proposition. Let $M$ be a complex curve (i.e. $n=1$ ). Then $F$ is a norm induced from a Hermitian metric with Gaussian curvature 
$\kappa$ if and only if the Legendre foliation on $S_{F}^{*} M$ satisfies the structure equations:

$$
d \theta^{1}=-\eta \wedge \xi^{3}, \quad d \eta=\theta^{1} \wedge \xi^{3}, \quad d \xi^{1}=\kappa \eta \wedge \theta .
$$

The 1-forms $\left\{\eta, \theta^{1}, \xi^{1}\right\}$ form an invariantly defined, global coframe on $S_{F}^{*} M$.

3.7 Lemma. The identity $\Pi(Z, Z)=1$ holds.

Proof: Recall that $Z=-J_{b}^{a} u^{b} \frac{\partial}{\partial u^{a}}$. By differentiating the first iderytity in $(2.10)$ with respect to $\frac{\partial}{\partial u^{c}}$, we obtain $J_{c}^{a} F_{a}+J_{b}^{a} u^{b} F_{n c}=0$. Using these, we computc

$\Pi(Z, Z)=F_{a b} J_{p}^{a} u^{p} J_{q}^{b} \eta^{q}=-J_{l}^{\prime 2} F_{a} J_{q}^{b} u^{q}=F_{a} u^{a}=F=1$ on $S_{f}^{*} M$

By the lemma, we can find a local frame $\left(Z_{\alpha}\right)$ of $L$ such that $Z_{1}=$ $Z$, along with a coframe $\left(\theta^{\alpha}, \eta, \xi^{\beta}\right)$ satisfying the condition (3.3) and the structure cquation (3.4). Let $\left(X_{\alpha}, X, Z_{\beta}\right)$ denote the dual frame of $\left(\theta^{\alpha}, \eta, \xi^{\beta}\right)$.

3.8 Lernma. The vector fields $J X$ and $[X, J X]$ have the following expressions in terms of the frame $\left(X_{\alpha}, X, Z_{\beta}\right)$ :

$$
[X, J X]=2 \pi_{1}^{\alpha}(X) X_{r x}+\left\{X\left(\pi_{1}^{\alpha}(X)\right)+\pi_{\beta}^{\alpha}(X) \pi_{1}^{\beta}(X)-S_{1}^{\alpha}\right\} Z_{\alpha} .
$$

Proof: To prove the lemma, note that the vector $J X$ and $[X, J X]$ has no component in the direction of $X$ since $\eta(J X)=\eta(|X, J X|)=0$ (recall from Emmin $(2.13)$ that $\zeta(J X)=\zeta([X, J X])=0)$. To compute the components in the other directions, we will make use of the fact that the Lic derivative $\mathcal{L}$ is a derivation and that the forms $\theta^{\prime}, \xi^{\alpha}$ annihilate $X$ Using the identity $J X=-\mathcal{L}_{2} X$ (Lemma 2.11) and the structure equation (3.4), compute

$$
\begin{aligned}
\theta^{\alpha}(J X) & \left.\left.=\theta^{\alpha}\left(-\mathcal{L}_{Z} X\right)=\left(\mathcal{L}_{Z} \theta^{\alpha}\right)(X)=\{Z\lrcorner d \theta^{\alpha}+d(Z\lrcorner \theta^{\alpha}\right)\right\}(X) \\
& =d \theta^{\alpha}(Z, X)=\delta_{1_{\alpha}} \\
\xi^{\alpha}(J X) & \left.\left.=\xi^{\alpha}\left(-\mathcal{L}_{Z} X\right)=\left(\mathcal{L}_{Z} \xi^{\alpha}\right)(X)=\{Z\lrcorner d \xi^{\alpha}+d(Z\lrcorner \xi^{\alpha}\right)\right\}(X) \\
& =d \xi^{\alpha}(Z, X)=\pi_{\beta}^{\alpha}(X) \xi^{\beta}(Z)=\pi_{1}^{\alpha}(X) .
\end{aligned}
$$


This proves the identity (3.9). To compute the component of $[X, J X]$ in the direction of $X_{\alpha}$, note that the identity (3.9) implies $\theta^{\alpha}(J X)=0$ and compute

$$
\begin{aligned}
\theta^{\alpha}([X, J X]) & =\theta^{\alpha}\left(\mathcal{L}_{X}(J X)\right)=X\left(\theta^{\alpha}(J X)\right)-\left(\mathcal{L}_{X} \theta^{\alpha}\right)(J X) \\
& \left.\left.=-\{X\lrcorner d \theta^{\alpha}+d(X\lrcorner \theta^{\alpha}\right)\right\}(J X)=d \theta^{\alpha}(J X, X)
\end{aligned}
$$

Using this identity, the structure equation (3.4) and the identity (3.9) again, we compute the component of $[X, I X]$ in the direction of $X_{\alpha}$ :

$$
\begin{aligned}
\theta^{\alpha}([X, J X]) & =d \theta^{\alpha}(J X, X) \\
& =\pi_{\beta}^{\alpha}(X) \theta^{\beta}(J X)+\xi^{\alpha}(J X)=\pi_{1}^{\alpha}(X)+\pi_{1}^{\alpha}(X)=2 \pi_{1}^{\alpha}(X) .
\end{aligned}
$$

In a similar manner, we compute the component of $[X, J X]$ in the direction of $Z_{\alpha}$ :

$$
\begin{aligned}
\xi^{\alpha}(\{X, J X]) & =\xi^{\alpha}\left(\mathcal{L}_{X}(J X)\right)=X\left\{\xi^{\alpha}(J X)\right\}-\left(\mathcal{L}_{X} \xi^{\alpha}\right)(J X) \\
& \left.\left.=X\left\{\xi^{\alpha X}(J X)\right\}-\{X\lrcorner d \xi^{\alpha}+d(X\lrcorner \xi^{\alpha}\right)\right\}(J X) \\
& =X\left\{\pi_{1}^{\alpha}(X)\right\}+d \xi^{\alpha}(J X, X) \\
& =X\left\{\pi_{1}^{\alpha}(X)\right\}+\pi_{\beta}^{\alpha}(X) \xi^{\beta}(J X)+S_{\beta}^{\alpha} \theta^{\alpha}(J X) \\
& =X\left\{\pi_{1}^{\alpha}(X)\right\}+\pi_{\beta}^{\alpha}(X) \pi_{1}^{\beta}(X)-S_{1}^{\alpha} .
\end{aligned}
$$

From these, the identity (3.10) follows.

\section{The Main Theorem}

4.1 Equivalent Conditions. In this scction, we state the nocessary and sufficient conditions for a complex Finsler manifold to satisfy the property (1.5). For each $v \in T_{x} M$, let $U_{v}$ be a neighborhood of 0 in the complex lire $\mathbb{C} \cdot v \subset T_{x} M$ spanned by $v$, on which the exponeritial map $\operatorname{cxp}$ is defined. The image $\exp \left(U_{x}\right)$ defines a surface in $M$ ncar $x$.

4.2 Theorem. The following conditions are equivalent.

Al. $\exp \left(U_{v}\right)$ is a complex curve for all $v \in T M$ with $\tilde{F}(v)=1$.

A2. $[X, J X]=\kappa Z$ on $S_{F}^{*} M$ for some snooth function $\kappa$.

A3. The distribution $\mathcal{D}$ spanned by $X, I X$ and $Z$ is an involutive distribution on $S_{F}^{*} M$.

Proof: We will prove the theorem by showing (i) the equivalence of conditions $\mathrm{Al}$ and $\mathrm{A} 2$, and (ii) the cquivalence of the conditions $\mathrm{A} 2$ and A3. 
(i) To prove the cquivalence of the conditions $\mathrm{A} 1$ and $\mathrm{A} 2$, recall (see Lemma 2.22) the identity

$$
\exp \left(t e^{y i} v\right)=\left\{\pi \circ e^{t X} \circ e^{-s Z}\right\}(\Phi(v)) .
$$

Thus, if we denote $\Gamma_{w}$ for the surface defined by $(t, s) \mapsto C_{w}(t, s)=$ $\pi\left(e^{t X} e^{-s Z} w\right)$ for $w \in S_{F}^{*} M$ and $t>0$ small, condition $A 1$ is equivalent to the condition that $\Gamma_{w}$ be a complex curve for all $w \in S_{F}^{*} M$.

Suppose that the condition A1 holds. Then the surface $\Gamma_{w}$ is a complex curve for all $w \in S_{i}^{*} M$. To show that the condition A2 holds, we show $[X, J X]=\kappa Z$ at $\tilde{w}=e^{\epsilon X} w \in S_{F}^{*} M$ for sufficiently strall $\epsilon>0$ and $w$ chosen arbitrarily in $S_{F}^{*} M$. Let $\left\{\tilde{\Gamma}_{\tau}\right\}$ denote a farrily of surfaces parametrized by $\tau$ defined by the function

$$
(t, s) \mapsto \tilde{C}_{r}(t, s) \equiv \pi\left(e^{(t-\tau) X} e^{s} e^{\tau X} u\right) .
$$

Observe that $\tilde{\Gamma}_{\tau}{ }^{1}$ s are complex curves since $\bar{C}_{\tau}(t, s)=C_{c ; \tau} X_{w}(t-\tau, s)$. Also, $C$ and $\tilde{C}_{\tau}$ coincide when $\tau=0$. Morcover, since $\tilde{C}_{\tau}(t, 0)=C_{w}(t, 0)$ for any $\tau \in[0, \epsilon]$, the surfaces $\tilde{\Gamma}_{\tau}$ contain the curve $C_{w}(t, 0)$ in commort, and in particular, the curves $\vec{C}_{\tau}(t, 0)$ have common end point $\pi(\dot{w})$ ind common tangent vector $T=\pi_{*} X_{\dot{u}}$ at the point $\pi(\tilde{w})$ when $t=\epsilon$. Sinec $\tilde{C}_{\tau}$ are complex curves, it follows that they have common tangent space: $\mathbb{C} \cdot T$ at $\pi(\tilde{w})$.

Let $W(\tau)$ be the tangent vector at $\pi(\tilde{u})$ defined by

$$
W(\tau)=\frac{\partial \tilde{C}_{\tau}}{\partial s}(\epsilon, 0)
$$

Then, $W(\tau) \in \mathbb{C} T$ for all $\tau \in[0, \epsilon]$, and therefore, all the derivatives of $W(\tau)$ are in $\mathbb{C} \cdot T$. Using the definition of Lie derivative, we compute the, derivatives of $W(T)$ :

$$
\begin{aligned}
& W(\tau)=\pi_{*} e_{*}^{(c-\tau) X} Z_{\left(e^{*} x w\right)}, \quad \frac{d W}{d \tau}=\pi_{*} e^{(c-\tau) X}\left\{\mathcal{L}_{X} Z\right\}_{\left(e^{\tau} \times(w)\right.}
\end{aligned}
$$

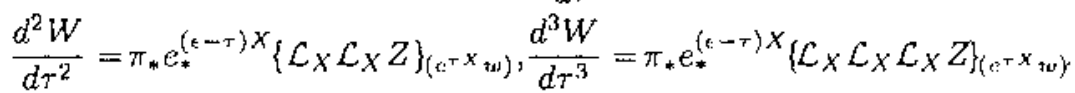

If we let. $\tau=\epsilon$, we obtain

$$
\pi_{*}\left(\mathcal{L}_{X} Z\right)_{\bar{u}} \in \mathbb{C} T, \quad \pi_{*}\left(\mathcal{L}_{X} \mathcal{L}_{X} Z\right)_{\bar{\nu}} \in \mathbb{C} T, \quad \pi_{*}\left(\mathcal{L}_{X} \mathcal{L}_{X} \mathcal{L}_{X} Z\right)_{\bar{u}} \in \mathbb{C} T .
$$

Since $T=\pi_{*} X_{\dot{t}}$ and $\mathcal{L}_{X} Z=J X=X_{1}+\pi_{1}^{\alpha}(X) Z_{\alpha,}$ we can conchude that

$$
[X, J X]_{\bar{u},}, \quad\left(\mathcal{L}_{X}[X, J X)_{\bar{w}} \in \mathbb{R} X_{\bar{w}} \oplus \mathbb{R}\left(X_{1}\right)_{\bar{w}} \ominus L_{\bar{w}} .\right.
$$


But from Lemma $3.8,[X, J X]$ does not have a component in the direction of $X$ and $X_{1}$, and thus $[X, J X]_{\tilde{w}} \in L_{\tilde{w}}$. Again, from Lemma 3.8, it follows that $\pi_{1}^{\alpha}(X)=0$, and we obtain

$$
J X=X_{1}, \quad \text { and } \quad[X, J X]=S_{1}^{\alpha} Z_{\alpha} .
$$

From the structure equation (3.6), it can be easily shown that the identity $\mathcal{L}_{X} Z_{\alpha}=X_{\alpha}+\pi_{\beta}^{\alpha}(X) Z_{\beta}$ holds. This identity and the second identity of (4.3) gives

$$
\begin{aligned}
\mathcal{L}_{X}[X, J X]=\mathcal{L}_{X}\left(S_{1}^{\alpha} Z_{\alpha}\right. & =\left(X S_{1}^{\alpha}\right) Z_{\alpha}+S_{1}^{\alpha}\left(\mathcal{L}_{X} Z_{\alpha}\right) \\
& =\left\{\left(X S_{1}^{\alpha}\right) Z_{\alpha}+S_{1}^{\beta} \pi_{\beta}^{\alpha}(X)\right\} Z_{\alpha}+S_{1}^{\alpha} X_{\alpha} .
\end{aligned}
$$

But, again, since we proved $\left(\mathcal{L}_{X}[X, J X]\right)_{\bar{w}} \in \mathbb{R} X_{\bar{w}} \mathbb{D} \mathbb{R}\left(X_{1}\right)_{\bar{w}} \ominus L_{\bar{w}}$, components of the vector $\mathcal{L}_{X}[X, J X]$ in the direction of $X_{\alpha}$ for $\alpha \neq 0$ has to vanish. Therefore, it follows that $S_{1}^{\alpha}=0$ for $\alpha>1$ and $[X, J X]=$ $S_{1}^{1} Z$, and the condition A2 follows.

To prove the converse, suppose that $[X, J X]=\kappa Z$ holds for some $\kappa \in C^{\infty}\left(S_{F}^{*} M\right)$. We prove that the surface defined by $C_{u}(t, s)=$ $\pi\left(e^{i X} e^{-s z} w\right)$ is a complex curve for all $w \in T^{*} M$ by showing that $J \frac{\partial C_{w}}{\partial t}(-\tau, 0)$ is thingent to the curve $C_{w}$ for small $r<0$. Note that $\mathcal{L}_{X} \mathcal{L}_{X} Z=\mathcal{L}_{X}[X, Z]=\mathcal{L}_{X}(J X)=\kappa Z$, and let. $\tilde{w}=e^{-\tau X} w$. Using the definition of the Lie derivative, we complute

$$
\begin{aligned}
\frac{d^{2}}{d t^{2}}\left(e_{*}^{-t X} Z_{\left(e^{2 X} \bar{w}\right)}\right) & =\frac{d}{d t}\left(e_{*}^{-t X}\left\{\mathcal{L}_{X} Z\right\}_{\left\{c^{t} x_{\tilde{w}}\right)}\right) \\
& =e_{*}^{-t X}\left\{\mathcal{L}_{X} \mathcal{L}_{X} Z\right\}_{\left(e^{t X} \bar{w}\right)} \\
& =\kappa\left(e^{t X} \bar{w}\right)\left\{e^{-t X} Z_{\left\{e^{t X} \bar{w}\right)}\right\} .
\end{aligned}
$$

Therefore, if wc let $W(t)=e_{*}^{-l X} Z_{\left(e^{t x}, \dot{w}\right)} \in T_{\tilde{w}}\left(S_{F}^{*} M\right), W(t)$ satisfies a second order ordinary differential equation $W^{\prime \prime}(t)=\kappa(t) W(t)$ with initial conditions $W(0)=Z_{\bar{w}}$ and $W^{\prime}(0)=J X_{\bar{w}}$. Consequently, we have $W(t) \in \operatorname{span}\left\{Z_{i \tilde{i}}, J X_{\tilde{w}}\right\}$ for small $t$, and in particular, $W(\tau)=$ $e_{*}^{-\tau X} Z_{\left(e^{\tau X} \bar{w}\right)} \in \operatorname{spari}\left\{Z_{\bar{w}}, J X_{\bar{w}}\right\}$. Substituting $\tilde{w}=e^{-\tau X} w$;

$$
W(\tau)=e_{*}^{-\tau X} Z_{w} \in \operatorname{span}\left\{Z_{\tilde{w}}, J X_{\bar{w}}\right\} .
$$

Using the identity $\frac{\partial C_{w}}{\partial t}(\tau, 0)=\pi_{*} X_{\tilde{w}}$ and $\pi_{*} \circ J=J \circ \pi_{*}$, we obtain

$$
\begin{aligned}
\frac{\partial C_{w}}{\partial s}(\tau, 0) & =\left.\frac{\partial}{\partial s}\right|_{s=0}\left\{\pi\left(e^{\tau X} e^{s Z} w\right)\right\} \\
& =\pi_{*} e_{*}^{\tau X} Z_{w}=k \pi_{*}(J X)_{\bar{w}}=k J \pi_{*}\left(X_{\dot{w}}\right)=k J \frac{\partial C_{w}}{\partial t}(\tau, 0)
\end{aligned}
$$


for some $k \in \mathbb{R}$, and hence $J \frac{\partial C_{w}}{\partial t}(\tau, 0)$ is tangent to the surface defined by $C_{w}$.

(ii) If $[X, J X]=\kappa Z$ holds for some $\kappa \in C^{\infty}\left(S_{F}^{*} M\right)$, then the involutivity of $\mathcal{D}$ follows from the identities

$$
[Z, X]=-J X, \quad[Z, J X]=X \quad \text { and } \quad[X, J X]=\kappa Z .
$$

The converse of this is an immediate consequence of the the identity (3.10).

\subsection{Remark.}

(1) Note that, in the proof of Theorem 4.2 , the two equations $\pi_{1}^{\alpha}(X)=$ 0 and $S_{1}^{\beta}=0$ for $\beta>1$ are equivalent to the single condition $[X, J X]=\kappa Z$ with $\kappa=S_{1}^{1}$. The condition $\pi_{1}^{\alpha}(X)=0$ can be interpreted as a compatibility condition for $F$ and the complex structure. For example, if $F$ is the induced norm of a Kachler manifold, this condition is satisfied. In this case, the condition $S_{1}^{\beta}=0$ for $\beta>1$ puts restrictions on the curvature of the Kaehler metric. One special case of this is when $M$ is a Kaehler manifold with constant holomorphic sectional curvature. In this case, it can be verified using results in [P] that $S_{1}^{\beta}=c \delta_{1}^{\beta}$ for some constant $c$.

(2) Conditions equivalent to AI-A.3 of Theorem 4.2 were introduced by Royden $[\mathbf{R}]$ and Faran $[\mathbf{F}]$.

The conditions AI-A3 in Theorem 4.2 can be equivalently stated as conditions on $T_{0}^{*} M$.

4.7 Theorem. The following conditions are equivalent:

B1. exp $\left(U_{v}\right)$ is a complex curve for all $v \in T_{0} M$.

B2. $[X, J X]=\kappa Z$ on $T_{0}^{*} M$ for some $\kappa \in C^{\infty}\left(T_{0}^{*} M\right)$.

B3. The distribution $\mathbb{C} X \oplus \mathbb{C} Z \subset T\left(T_{0}^{*} M\right)$ is involutive.

Moreover, these conditions are equivalent to conditions A1-A3 in Theorem 4.2 .

Proof: Note that the conditions A1 and B1 are clearly equivalent. To prove the theorem, we show (i) that $A 2$ implies $B 2$, and (ii) that $A 3$ implies B3. The converses of these are trivial to prove.

(i) Suppose that $[X, J X]=\kappa Z$ holds on $S_{F}^{*} M$ for some $\kappa \in C^{\infty}\left(S_{F}^{*} M\right)$. Extend $\kappa$ to $T_{0}^{*} M$ by $\kappa(t w)=t^{2} \kappa(w)$ for all $t>0$ and $w \in S_{F}^{*} M$.

We claim that $[X, J X]=\kappa Z$ on $T_{0}^{*} M$. To show this, we show that both $W=[X, J X]$ and $W=\kappa Z$ on $T_{0}^{*} M$ must satisfy the ordinary differential equation $\mathcal{L}_{Y} W=2 W$. Note that the integral curves of $Y$ 
are the radial lines in $T_{0}^{*} M$. Thus, if both $[X, J X]$ and $\kappa Z$ satisfy the equation, they inust coincide because the identity $[X, J X]=\kappa Z$ gives the same initial condition at points on $S_{F}^{*} M$. To show that the vector ficld $[X, J X]$ satisfics the differential equation, recall, from Lemma 2.11, that $\mathcal{L}_{Y} X=X$ and $\mathcal{L}_{Y} J X=J X$. Using these identities and the fact, that $\mathcal{L}_{Y}$ is a derivation, we compute

$\mathcal{L}_{Y}[X, J X]=\left[\mathcal{L}_{Y} X, J X\right]+\left[X, \mathcal{L}_{Y} J X\right]=[X, J X]+[X, J X]=2[X, J X]$.

To show that the vector field $\kappa Z$ satisfies the differential equation, recall that the vector field $Y$ on $T_{0}^{*} M$ is generated by the action of $\mathbb{R}$ by multiplication of $e^{t}$. Using homogeneity of $\kappa$, we obtain the following identity: For $u \in T_{i}^{*} M$,

$$
(Y \kappa)(w)=\left.\frac{d}{d t}\right|_{t=0} \kappa\left(e^{\imath} u\right)=\left.\frac{d}{d t}\right|_{t=0}\left\{e^{2 \ell} \kappa(w)\right\}=2 \kappa(w)
$$

Using this identity and the fact that the vector fields $Y$ and $Z$ commute (i.e. $\mathcal{L}_{Y} Z=[X, Y]=0$ ), we compute

$$
\left\{\mathcal{L}_{Y}(\kappa Z)\right\}=(Y \kappa) Z=2(\kappa Z)
$$

(ii) The proof that the condition A3 inplies the condition B3 immediately follows from the identities in Lemma 3.8 .

4.8 Totally Geodesic Complex Curves. We call a complex curve $\Sigma$ totally geodesic if, for any tangent vector $v$ to the complex curve $\Sigma$ and geodesic segment $\gamma_{v}:(-\epsilon, \epsilon) \longrightarrow M$ such that $\frac{d \gamma_{v}}{d t}(0)=v, \gamma_{v}(t)$ is contained in the complcx curve for small $t$. The main result of this section is that, under condition (1.5), the geodesics of $F$ can be uniquely extended to irnmersed complex curves that are totally gcodesic submanifolds of $M$. In fact, these curves are precisely the oncs defined by the complex curves exp $\left(U_{v}\right)$ 's in condition (1.5).

Theorem 4.9. If the condition (1.5) holds, the complex curve $\exp \left(U_{y}\right)$ can be uniquely extended to a maximal totally goodesic complex. curve $f: \Sigma \hookrightarrow M$ immersed in $M$. Moreover, there is a canonical $(n-1)$ dimensional holomorphic vector subbundle $T^{\perp} \Sigma$ of $f^{*}(T M)$ transversal to $\Sigma$.

Proof: Rocall from Theorem 4.2 that $\mathcal{D}=\operatorname{span}\{X, J X, Z\}$ is an involutive distribution. By the Frobenius theorcm, this implics that $S_{F}^{*} M$ is foliatcd by 3-dimcrisional maximal integral subrmanifolds of $\mathcal{D}$. Let 
$\tilde{\Sigma}$ be a leaf of this foliation $\mathcal{F}_{\mathcal{D}}$, then there is a well defined $S^{1} \subset \mathbb{C}$ action on $\tilde{\Sigma}$ since $Z$ is tangent to $\tilde{\Sigma}$. Let us denote the quotient space $\tilde{\Sigma} / S^{1}$ by $\Sigma$. It is not difficult to see that $\Sigma$ is a complex curve with the complex structure induced from the complex structure of $\tilde{\Sigma} \subset T^{*} M$, and that there is a holomorphic inmersion $f$ such that the following diagram commutes:

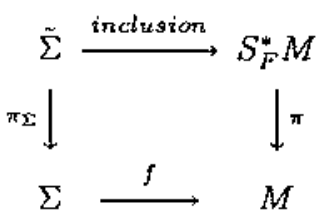

Recall that the complex curve $\exp \left(U_{\nu}\right)$ is the surface defined by

$$
(t, s) \mapsto \exp \left(t e^{s z} v\right)=\pi\left(e^{\imath X} e^{-s z} \Phi(v)\right)
$$

From this, it is clear that the complex curve $f: \Sigma \rightarrow M$ is locally defined by $\exp \left(U_{v}\right)$ since $e^{\ell X} e^{-s Z} w \in \Sigma$. From this, it clcarly follows that $f: \Sigma \rightarrow M$ is totally gcodesic.

To define the transversal holomorphic subburdle $T^{\perp} \Sigma$ of $f^{*}(T M)$, note that $\pi_{\Sigma}: \tilde{\Sigma} \rightarrow \Sigma$ is a principal circle bundle. Define the fiber $T_{T}^{1} \Sigma$ of $T^{\perp} \Sigma$ at $x \in \Sigma$ by $T_{x}^{\perp} \Sigma=\left\{v \in f^{*}(T M) \mid w\left(f_{*} v\right)=0\right.$ for all $\left.w \in \tilde{\Sigma}_{x}\right\}$. It is clear that $T^{\perp} \sum$ is a holomorphic vector bundle with dimersion $n-1$.

To show that $T_{x}^{\perp} \Sigma$ is transversal to $\Sigma$, suppose $v \in T^{\perp} \Sigma \cap T \Sigma$. Note that $\Psi(w)$ is tangent to $\Sigma$ for all $w \in \tilde{\Sigma}_{x}$ since $\Psi(w)=\pi_{*} X_{w}$ and $X$ is tangent to $\tilde{\Sigma}$. Moreover, $\Psi(w) \neq 0$ becaluse $w(\Psi(w))=w\left(\pi_{*} X_{w}\right)=$ $F F_{a} u^{a}=F^{2}=1 \neq 0$, where $u=\sum_{a=1}^{n} u^{a} d x^{a}$. Since $v, f^{*} \Psi(w) \in T_{x} \Sigma$, we have $f_{*} v=z \Psi(u)$ for some $z \in \mathbb{C}$. But, recall that $v \in T^{\perp} \Sigma$, and therefore, $w(v)=0$. This implies that $v=0$ because

$$
w(v)=w(z \Psi(w))=z\{w(\Psi(w))\}=z .
$$

4.12 The Holomorphic Sectional Curvature. If $F$ satisfies the property ( 1.5$)$, there is a natural way to define holomorphic sectional curvature $K$ of $F$. In this section, we show that $K$ is determined by the smooth function $\kappa$ in the condition A2 of Theorem 4.2.

Holomorphic sectional curvature $K$ of a complex Finsler metric $F$ has been studied by Wong and Royden [W] [R]. To definc $K(v)$ for a unit vector $v \in T_{x} M$ (i.c. $\tilde{F}(v)=1$ ), note that each complex curve $U \subset M$ tangent to $v$ has a canonical complex Finsler metric defined by the restriction $\tilde{F}_{\mid T U}: T U \rightarrow \mathbb{R}$. In fact, bccausc $U$ is of complex 
dimension one, it can be casily seen that the metric $\tilde{F}_{\mid T U}$ is a norm induced by a Hermitian metric $g$ on $U$ : If $\tilde{F}(v)=1$,

$$
\bar{F}((\alpha+i \beta) v)=|\alpha+i \beta| \bar{F}(v)=\sqrt{\alpha^{2}+\beta^{2}} .
$$

In $[\mathbf{W}]$, Wong defined the holomorphic sectional curvature $K(v)$ as the supremum of the Gaussian curvature of $g$ at $x \in U$, where supremum is taken over all complex curves tangent to $v$. In the special case when $F$ is the norm induced by a Hcrmitian metric, this defines the usual holomorphic sectional curvature of Hermitian metric. Observe that, if $F$ satisfies the property (1.5), then by Theorem 4.2 , there is a naturally defined totally geodesic complex curve of the form exp $\left(U_{v}\right)$ tangent to $v$. In [R], Royden showed that the Gaussian curvature of the induced metric $g$ at $x$ on this complex curve attains the grcatest value and, hence, it defines the holomorphic sectional curvature $K(v)$. Thus, the following theorem holds:

4.13 Theorem. Let $N$ be a complex submanifold of $M$, and let $K^{\prime}$ be the holomorphic sectional curvature of the induced metric: $\bar{F}_{\mid T N}$ on $N$. Then the inequality

$$
K^{\prime}(v) \leq K(v)
$$

holds every unit vector $v$ tangent to $N$.

In particular, if $U \subset M$ is a complex curve tangent to a unit vector $v \in T M$, the Gaussian curvature of the induced metric $g$ defined by $\tilde{F}_{\mid T U}$ is bounded from above by $K(v)$.

The function $\kappa$ can be regarded as a function on the quotient space $S_{F}^{*} M / S^{1}$. Recall from $(2.12)$ and $(4.5)$ the identities $[Z, X]=-J X$, $[Z, J X]=X$ and $\kappa Z=[X, J X]$, and compute

$$
\begin{aligned}
(Z \kappa) Z & =\mathcal{L}_{Z}(\kappa Z)=\mathcal{L}_{Z}[X, J X] \\
& =\left[\mathcal{L}_{Z} X, J X\right]+\left[X, \mathcal{L}_{Z}(J X)\right]=[-J X, J X]+[X, X]=0 .
\end{aligned}
$$

From this identity, we conclude $Z \kappa=0$. This implies that the function $\kappa$ is invariart under the circle action of unimodular complex numbers defined by multiplication on $T_{0}^{*} M$, and hence the function $\kappa$ can be regarded as a function on $S_{F}^{*} M / S^{\dagger}$.

4.14 Theorem. If $K$ is the holomoryhic sectional curvatare of $F$,

$$
K(v)=\kappa \circ \Phi(v)
$$

for every unit vector $v \in T M$.

Bcfore we begin the proof, note that by Theorem 4.9 the complex curve $U \subset M$ has a unique extension to a maximal totally geodesic complex curve $f: \Sigma \rightarrow M$, and that therc is a circle bundle $\pi_{\Sigma}: \tilde{\Sigma} \rightarrow \Sigma$ over $\Sigma$. 
4.15 Lemma. The fibers of the circle bundle $\pi_{\Sigma}: \tilde{\Sigma} \longrightarrow \Sigma$ defines a Legendre foliation $\mathcal{F}_{\bar{\Sigma}}$ with respect to a natural contact, 1-form defined by the pull-back of $\eta$ to $\Sigma$. The structure equations of this Legendre foliation are the pull backs to $\Sigma$ of the equations:

$$
d \theta^{1}=-\eta \wedge \xi^{1}, \quad d \eta=\theta^{1} \wedge \xi^{1}, \quad d \xi^{1}=\kappa \eta \wedge \theta^{1}
$$

Proof: The structure equations (4.16) are obtaincd by pulling back the equation (3.4) to $\tilde{\Sigma}$. Note that, since $J X=X_{1}$ and $Z=Z_{1}$, we have $\theta^{\alpha}(J X)=\xi^{\alpha}(Z)=0$ for $\alpha>1$. Therefore, on $\dot{\Sigma}$, we have

$$
\begin{aligned}
d \theta^{1} & =-\eta \wedge \xi^{1}+G^{1}{ }_{11} \theta^{1} \wedge \xi^{1} \\
d \eta & =\theta^{1} \wedge \xi^{1} \\
d \xi^{1} & =S_{1}^{1} \eta \wedge \theta^{1}+Q^{1}{ }_{11} \theta^{1} \wedge \xi^{1} .
\end{aligned}
$$

But, from the identities (4.5), it easily follows that $G^{1}{ }_{11}=Q^{1}{ }_{11}=0$ and $S_{1}^{1}=\kappa$. Hence, we obtain the structure cquations (4.16):

From the equations, it is clear that the pull back to $\Sigma$ of $\eta$ is a contact, form since $\eta \wedge d \eta=\eta \wedge \theta^{1} \wedge \xi^{1} \neq 0$ on $\tilde{\Sigma}$.

Proof: To prove the theorem, choose $v \in T_{x} M$ and let $U \subset M$ be a totally geodesic cornplex curve such that $x \in U$ and $v \in T_{x} U$. Also, let $f: \Sigma \rightarrow M$ be the unique extension of $U$ described in Theorem 4.9, and let $\tilde{\Sigma}_{U}$ denote the restriction of the circle bundle $\pi_{\Sigma}: \Sigma \rightarrow \Sigma$ to $U$. Observe that $U$ is also a submanifold of $S_{i}^{*} M / S^{1}$ since $U \subset \Sigma=$ $\Sigma / S^{1} \subset S_{F}^{*} M / S^{1}$. We claim that the Gaussian curvature of $g$ on $U$ is $\kappa_{\mid U} \in C^{\infty}(U)$, where $\kappa$ is regarded as a function on $S_{\%}^{*} M / S^{1}$.

The theorem follows from the clairn. To see this, proceed as follows: Note that by commutativity of the diagram (4.10), $\pi=f \circ \pi_{\Sigma}$. Hernee, if $w \in \tilde{\Sigma}_{U}$, then $\Psi(w)=\pi_{*} X_{w}=f_{*} \circ\left(\pi_{\Sigma}\right)_{*} X_{v}$. Therefore, the map $\Psi: S_{F}^{*} M \rightarrow S_{F} M$ sends $\Sigma_{U}$ into the unit tangent bundle $S_{g} U$ of $g$. Since $\Psi$ is a bundle map over $U$ such that $\Psi\left(e^{s i} w\right)=e^{-s i} \Psi(w)$, $\Psi$ mans $\Sigma_{U}$ diffeomorphically onto $S_{g} U$, or equivalently, we have a bundle rrap $\Psi_{\mid S_{g} U}^{-1}=\Phi_{\mid S_{g} U}: S_{g} U \rightarrow \dot{\Sigma}_{U}$ over $U$. Thus, since $\kappa$ is constant along fibers of $\tilde{\Sigma}_{U}$ and $v \in T_{x} U$, we have $\kappa \circ \Phi(v)=\kappa(x)$. By the claim, $\kappa(x)$ is the Gaussian curvature of $g$ at $x \in U$, and the identity $K(v)=\kappa .0 \Phi(v)$ follows.

To prove the claim, we denote the Gaussian curvature of $g$ on $U$ by $\bar{\kappa} \in C^{\infty}(U)$, and show $\kappa=\vec{\kappa}$. Recall that, from Proposition 3.5, the Legendre foliation on $S_{9}^{*} U$ has the structure cquations

$$
d \tilde{\theta}^{1}=-\vec{\eta} \wedge \tilde{\xi}^{1}, \quad d \tilde{\eta}=\tilde{\theta}^{1} \wedge \tilde{\xi}^{1}, \quad d \tilde{\xi}^{1}=\bar{\kappa} \tilde{\eta} \wedge \tilde{\theta}^{1},
$$


where $\left\{\tilde{\theta}^{1}, \tilde{\eta}, \tilde{\xi}^{1}\right\}$ is the invariant coframe on $S_{9}^{*} U$. The proof of the claim is done by establishing the cquivalence of the Legendre foliation on $S_{g}^{*} U$ with $\mathcal{F}_{\mathfrak{\Sigma}}$. This is proved by showing that there is a diffeomorphism $\psi: \tilde{\Sigma}_{U} \rightarrow S_{9}^{*} U$ such that

$$
\psi^{*} \tilde{\eta}=\eta, \quad \text { and } \quad \pi \circ \psi=\pi_{\Sigma}
$$

It follows that $\psi^{*} \bar{\theta}^{1}=\theta^{1}$ and $\psi^{*} \tilde{\xi}^{1}=\xi^{1}$, and in particular, the pull-back by $\psi$ of the equations (4.17) are the structure equations $(4.16)$ of $\mathcal{F}_{\bar{\Sigma}}$. The identity $\vec{\kappa} \circ \psi=\kappa$ follows.

We define $\psi(w)$ for $w \in \tilde{\Sigma}_{U} \subset T^{*} M$ as the by pull-back of $w$ to the tangent space of $U$. In coordinates, we have

$$
\left(x^{1}, 0, . ., 0, x^{n+1}, 0, \ldots, 0 ; u^{1}, \ldots, u^{n}, u^{n+1}, \ldots, u^{2 n}\right) \stackrel{\psi}{\longrightarrow}\left(x^{1}, x^{n+1}, u^{1}, u^{n+1}\right),
$$

where $\left(x^{1}, \ldots, x^{n}, x^{n+1}, \ldots, x^{2 n}\right)$ is taken so that $U \subset M$ is locally defined by $x^{n}=0$ for $a \neq 1, n+1$. To complete the proof, it remains to show that

(1) $\psi$ maps $\tilde{\Sigma}_{y}$ diffeomorphically onto $S_{g}^{*} U$, arid

(2) $\psi^{*} \tilde{\eta}=\eta$

(1): To show that $\psi$ is a diffeomorphism onto $S_{g}^{*} U$, recall that $\Psi$ maps $\tilde{\Sigma}_{U}$ onto $S_{g} U$. Hence, if $w \in \tilde{\Sigma}_{U}$, then the vectors $\Psi(w)$ and $\Psi(J w)=$ $-J \Psi(u)$ form an orthonormal frame of $T_{x} U$ for some $x \in U$. The following computation shows that the covectors $\psi(w)$ and $\psi(J w)=J \psi(w)$ form the dual coframe of $\{\Psi(m), \Psi(J u)\}$ : Using the identities (2.10), compute:

$$
\begin{aligned}
\{\psi(w)\}(\Psi(w)) & =w\left(\pi_{*} X_{w}\right)=F F_{a} u^{a}=F^{2}=1 \\
\{\psi(J w)\}(\Psi(J w)) & =\{J \psi(w)\}(-J \Psi(w))=\{\psi(w)\}(\Psi(w))=1 \\
\{\psi(w)\}(\Psi(J w)) & =\{\psi(w)\}(-J \Psi(w))=\{-J \psi(w)\}(\Psi(w)) \\
& =\{-J w\}\left(\pi_{*} X_{w}\right)=u^{a} J_{u}^{b} F F_{b}=0 .
\end{aligned}
$$

Hence $\psi\left(\tilde{\Sigma}_{U}\right) \subset S_{q}^{*} U$. Since $\psi$ is a bundle map preserving the circle action, it easily follows that $\psi$ is a diffeomorphism.

(2): To prove the identity $\psi^{*} \tilde{\eta}=\eta$, recall from (2.2) that $\tilde{\eta}=u^{1} d x^{2}+$ $u^{n+1} d x^{n+1}$. Therefore, from (4.19), $\psi^{*} \vec{\eta}=u^{1} d x^{1}+u^{n+1} d x^{n+1}$. On the other hand, the contact 1-form $\eta$ on $\Sigma$ is defined by the pull-back of $\eta=\sum_{a=1}^{2 n} u u^{a} d x^{2}$ to $\tilde{\Sigma}$. But, since $d x^{a}=0$ for $a \neq 1, n+1$ on $\tilde{\Sigma}$, we have $\eta=u^{1} d x^{1}+u^{n+1} d x^{n+1}$. Hence the identity $\psi^{*} \tilde{\eta}=\eta$ follows.

The following corollary is a consequence of the proof of Thoorem 4.14: 
4.20 Corollary. The Ganssian curvature of the induced metric $g \mathrm{~mm}$ $\Sigma$ is $\kappa_{\mid \bar{\Sigma}}$.

4.21 Relation to the Kobayashi Metric. In this section, we prove a version of a theorem of Faran which states that vanishing of certain local invariants forces $F$ to be the Kobayashi metric of $M$, provided that $F$ is complete and satisfies the condition $K=-4$ (sce Iritroduction). In the version of the theorcm prescnted here, the condition of vanishing of invariants is replaced by the equivalent condition (1.5).

Recall from Lempert's result described in the introduction that, if $D \subset \mathbb{C}^{n}$ is a bounded strictly linearly convex domain with smooth boundary, then every extremal disk $f: \Delta \rightarrow D$ is an isometric imbedding (i.c. $f^{*} F_{K}$ coincides with the Poincaré norm on $\Delta$ ), and that $f(\Delta)$ is a maximal totally geodesic complex curve in $D$. Sinec the Poincaré metric: has Gaussian corvature -4 , the holomorphic sectional curvature of the Kobayashi metric $F_{K}$ is -4 .

On the other hand, if $F$ is any complex Finsler metric: on a complex manifold $M$, the condition of constant holomorphic sectional envature $K=-4$ imposes ar restriction on the metric $F$. In fact, we show that, if $F$ is any complete complex Finsler metric with the proporties $K=-4$ and (1.5), thern $F$ must coineide with the Kobayashi metric. To show this, we need the following lemma due to Ahlfors $\{\mathbf{A}][\mathbf{K}]$ :

4.22 Generalized Schwarz Lemma. Let $(N, g)$ be a 1-dimensismal Hermitian manifold such that the Gaussian curvature is bounded alowe by a negative constant $-C$. For any holomorphic map $f: \Delta \rightarrow N$, the inequality

$$
\left\|f_{*} v\right\|_{g} \leq \frac{4}{C}\|v\|
$$

holds for all $v \in T \Delta$, whtere \|\|$_{a}$ is the norm on $N$ indated by $g$ ard \|\| denotes the norm defined by the Poincare metric on $\Delta$.

We call a complex Finsler metric $F$ complete if the geodesic vector field $X$ is complete (or equivalently, if every geodesic cun be extonded to a geodesic defired or all of $\mathbb{R}$ ).

4.24 Theorem [F]. Suppose $F$ is a complete complex Finsler metric: on a complex manifold $M$ unth constant holomerptic sectional curvature $K=-4$ satisfying the property (1.5). The dual metric $\dot{F}$ coincides unith the Kobayashi matric $F_{K}$ of $M$.

Proof: We verify the equality $F_{K}=\dot{F}$ by verifying the inequalities $F_{K} \leq \dot{F}$ and $\dot{F} \leq F_{K}$. 
(i) To show that the incquality $F_{K} \leq \vec{F}$ holds, ket $v \in T M$ and recall from Theorem 4.9 that there is an immersed totally geodesic complex curve $f_{1}: \Sigma \rightarrow M$ tangent to $v$. Since $F$ is a completc metric with $K=-4$ and $\Sigma$ is totally geodesic, the induced metric $g$ on $\Sigma$ defined by $f_{1}^{*} \bar{F}$ is a complete Kaehler motric with Gaussian curvature -4 (see Thcorem 4.14 and Corollary 4.20). Therefore, there is a holomorphic covering map $f_{2}: \Delta \rightarrow \Sigma$ which is a local isometry between the Poincaré metric and $g$ (sce chapter IX of $[\mathrm{KN}]$ ). By composing $f_{1}$ and $f_{2}$, we obtain a holomorphic map $f_{1} \circ f_{2}: \Delta \rightarrow M$ that is an isometric immersion with respect to the Poincaré metric and $\tilde{F}$ (i.e. $\left\{\left(f_{1} \circ f_{2}\right)^{*} \tilde{F}\right\}(u)=\|w\|$ ). Recall that the Kobayashi metric $F_{K}(v)$ is defined as the infirmum of $\left\|f^{*} v\right\|$ over all complex curve $f: \Delta \rightarrow M$ tangent to $v$. Hence, the inequality follows:

$$
F_{K}(v) \leq\left\|\left(f_{1} \circ f_{2}\right)^{*} v\right\|=F(v) \quad \text { for } \quad v \in T M .
$$

(ii) To prove the inequality $\tilde{F}(v) \leq F_{K}(v)$, note that, for each complex curve $f: \Delta \rightarrow M$ tangent to $v \in T M$, there is a Hermitian metric metric gf on $\Delta$ defined by $f^{*} \tilde{F}$. By Theorem 4.13 , the Gaussian curvature $\kappa_{f}$ is bounded above by -4 . Applying the Generalized Schwarz Lemma 4.22 to the identity map id $: \Delta \rightarrow\left(\Delta, g_{f}\right)$, we obtain the inequality $\left\{f^{*} \tilde{F}\right\}(w) \leq\|w\|$, or cquivalently, $\tilde{F}\left(f_{*} w\right) \leq\|w\|$ for all $u \in T \Delta$. In particular, this implies that $\tilde{F}(v) \leq\left\|f^{*} v\right\|$ for any complex curve tangent to $v$. Since $F_{K}(v)$ is the infmum of $\left\|f^{*} v\right\|$ over all such $f$, the inequality $\tilde{F}(v) \leq F_{K}(v)$ follows.

\section{References}

[A] L.V. Antrors, An extcnsion of Schwarz's lemma, Trans. of Amer. Math. Soc. 43 (1938), 359-364.

[BD] J. Bl,AND, T. DuChamp, Moduli for Pointed Convex Domains," Invent. Math., (to appear).

[F] J.J. FARAN, Hermitian Finsler Mctrics and the Kobayashi Metric, Journal of Differential Geometry 31, no. 3 (1990), 601-625.

[GF] I.M. GElmand, S.V. Fomin, "Colculus of Variations," Prentice-Hall, Inc., Englewood Clifis, 1963.

[X] S. Koß АYASII, "Hyperbolic Manifolds and Holomorphic Mappings," Marcel Dekker, Inc., New York, 1970.

[KN] S. Kobayasıl, K. NOMIZU, "Foundations of Differential Geometry," Vol. 1 and 2, John Wiley \& Sons, Inc., New York, 1963. 
[L1] L. LEMPERT, La metrique de Kobayashi et la representation des domains sur la boule, Bull. Soc. Math. France 109 (1981), 427-474.

[L2] L. LEMPER', Intrinsic Distances and Holomorphic Retracts, Complex Analysis and Applications 81 (1984), $341 \cdot 364$.

[L3] L. LEMPERT, Holomorphic invariants, normal forms, and the moduli space of convex domains, Annals of Math. 128 (1988), 4378.

[P] M. PANG, The Structure of Legendre Foliations, Trans. of Amer. Math. Soc. 320, no. 2 (1990), 417-455.

[Pa] G. PATrizio, Disques extremaux de Kobayashi et equation de Monge-Ampere complexe, C. R. Acad. Sci. Paris 305, Serie I (1987), 721-724.

[R] H.L. ROYDEN, Complex Finsler Metrics, Contemporary Mathematics, Proccedings of Summer Research Conference, Aug. 12-18, Amer. Math. Soc, 1984, pp. 119"124.

[S] S. Sternibrg, "Lectures on Differential Geometry," Prentice Hall, Englwood Cliffs, 1964.

[W] B. Wovg, On the Holomorphic Curvature of Some Intrinsic Metries, Proc. Amer. Math. Soc. 65, no. 1 (1977), 57-61.

\author{
Department of Mathematics \\ Box 1146 \\ Washington University \\ St, Louis, MO $63130-4899$ \\ U.S.A.
}

Rebut el 12 de Març de 1991 